\title{
INSTRUMENTOS QUE AVALIAM O VÍNCULO MATERNO E OS RISCOS DE ALTERAÇÃO NO DESENVOLVIMENTO INFANTIL
}

\author{
Livia Rocha Rolim \\ Universidade de Lisboa \\ Faculdade de psicologia \\ liviarrolim@hotmail.com \\ João Joaquim Freitas do Amaral \\ Universidade Federal do Ceará \\ Faculdade de Medicina \\ Álvaro Jorge Madeiro Leite \\ Universidade Federal do Ceará \\ Faculdade de Medicina
}

Fecha de Recepción: 22 Agosto 2019

Fecha de Admisión: 25 Septiembre 2019

\section{RESUMO}

Este estudo fez parte das atividades desenvolvidas no Mestrado de Medicina do Programa de saúde Materno Infantil da Universidade Federal do Ceará, no Brasil. Objetivo. Avaliar a consistência bibliográfica do inventário de percepção de vinculação materna (IPVM) e do Survey of Wellbeing of Young Children (SWYC), apontando informações de confiabilidade e validade sobre suas medidas. Métodos: Este é um estudo de revisão, realizado no período de fevereiro a dezembro do ano de 2017, nas bases dos periódicos CAPES, na Biblioteca Virtual em Saúde (LILACS, MEDLINE e IBECS), no Pubmed e Scielo. Resultados: Constatou-se que esses instrumentos investigativos são bastante necessários nas pesquisas que envolvem saúde pública, por serem úteis para identificação precoce de riscos na saúde da criança, como também identificar e fortalecer os vínculos familiares dos cuidados das crianças de zero a três anos. Conclusão: Concluiu-se que o SWYC é tido como um instrumento bastante útil para vigilância do desenvolvimento neuropsicomotor, socioemocional e de comportamento, sendo também um importante sinalizador do contexto de vida das crianças. 0 IPVM mostrou-se consistente para avaliar a vinculação das mães de crianças de seis a treze anos de idade, denotando elevada fidedignidade, contudo indicamos a necessidades de novas pesquisas com este instrumento no Brasil, a fim de possibilitar a sua validação. Os dois instrumentos se mostraram úteis na atenção primária, por serem de fácil acesso, viáveis, de baixo custo e fácil aplicação, além de possuírem eficácia técnica e poderem ser utilizados em larga escala.

Palavras-chave: desenvolvimento infantil; relações mãe-filho; comportamento infantil; apego; diagnóstico; comportamental.

\section{ABSTRACT}

Instruments that assess maternal bonding and the risks of changes in child development. This study was part of the activities developed at the Master of Medicine of the Maternal and Child Health Program 


\section{INSTRUMENTOS QUE AVALIAM O VÍNCULO MATERNO E OS RISCOS DE ALTERAÇÃO NO DESENVOLVIMENTO INFANTIL}

of the Federal University of Ceará, Brazil. Objective: To evaluate the bibliographic consistency of the Maternal Attachment Perception Inventory (MAPI) and the Survey of Wellbeing of Young Children (SWYC), indicating reliability and validity information about their measurements. Methods: This is a review study, conducted from February to December of 2017, in the bases of the CAPES journals, the Virtual Health Library (LILACS, MEDLINE and IBECS), Pubmed and Scielo. Results: It was found that these investigative instruments are very necessary in research involving public health, as they are useful for early identification of risks in child health, as well as identify and strengthen family ties of care for children from zero to three years. Conclusion: It was concluded that SWYC is considered a very useful instrument for surveillance of neuropsychomotor, socioemotional and behavioral development, and is also an important indicator of children's life context. The MAPI proved to be consistent to assess the attachment of mothers of children from six to thirteen years of age, indicating high reliability, however, we indicate the need for further research with this instrument in Brazil, in order to enable its validation. Both instruments proved to be useful in primary care because they are easily accessible, viable, inexpensive and easy to apply, as well as being technically effective and widely used.

Keywords: child development; mother-child relations; child behavior; attachment; screening; behavioral.

\section{INTRODUÇÃO}

Diante de inúmeros estudos sobre o desenvolvimento infantil, pode-se perceber a relevância das primeiras relações da criança para a base do seu desenvolvimento, sendo essencial para um desenvolvimento saudável que 0 cuidador se adapte as necessidades da criança, através da interação, afeto e cuidados (Eickmann, Emond \& Lima, 2016; Weitzman \& Wegner, 2015).

Diante disso uma vez que há instrumentos para mensurar o grau dessas relações e a influência no desenvolvimento infantil. A partir de um estudo bibliográfico com foco em dois instrumentos pretende-se apresentar os resultados obtidos a partir do objetivo de avaliar a consistência bibliográfica do inventário de percepção de vinculação materna (IPVM) e do Survey of Wellbeing of Young Children (SWYC), apontando informações de confiabilidade e validade sobre suas medidas.

Desse modo, a seguir serão apresentados os instrumentos e os estudos identificados na pesquisa, a metodologia e a discussão dos resultados

\section{Inventário de Percepção de Vinculação Materna (IPVM)}

0 Inventario de Percepção de Vinculação Materna (IPVM), foi traduzido para o português Brasileiro em 2011, do original Maternal Attachment Inventory (MAI), criado em 1994 para avaliar quantitativamente a percepção do sentimento da mãe acerca do vínculo com o seu filho (Boeckel, Wagner, Ritter, Sohne, Schein \& Grassi-Oliveira 2011). 0 MAl foi traduzido e utilizado em diversos países como: Brasil, China, Espanha, Turquia, Japão, Jordânia e Coréia do Sul (Allende, Fuente, Rivera, \& García 2016; Boeckel, Wagner, Ritter, Sohne, Schein \& Grassi-Oliveira 2011; Boztepe, Ay, Kerimo lu, \& Çınar, 2016; Chen, Sung, Chen, Chang, \& Lee 2013; Gharaibeh \& Hamlan 2011; Gürol \& Polat 2012; Sen \& Kavlak 2012; Serçeku \& Ba kale 2016; Shin \& Kim, 2007; Suetsugu, Honjo, Ikeda \& Kamibeppu, 2015; Zhang, Su, Yao, Wang, Dang, Ding, Zhu, Shao \& Li 2017).

A tradução para o português brasileiro, foi realizada por meio de seis juízes que possuem profundo conhecimento na área e completo domínio da língua inglesa, eles adaptaram e fizeram a análise semântica e conceitual de cada item do instrumento (Boeckel, Wagner, Ritter, Sohne, Schein \& Grassi-Oliveira 2011).

0 MAl foi desenvolvido para avaliar a percepção vincular entre mães e seus filhos de até um ano de idade. Pesquisadores observaram que esse instrumento é composto por três fatores, o primeiro seria relacionado aos sentimentos de amor e prazer da mãe no decorrer da interação com o filho, o segundo é relacionado as preocupações da mãe com a criança e o terceiro seria 0 conhecimento da mãe referente às necessidades do seu filho (Shin \& Kim 2007).

Já o IPVM foi traduzido e adaptado para avaliar a percepção do vínculo das mães de crianças de seis a treze anos de vida, sendo composto por dois fatores, um fator é relacionado a interação e afeto da mãe com a criança 
e 0 outro é referente a percepção da mãe em relação a sua vinculação com o filho. 0 IPVM se diferencia do original MAl que é composto por trinta e uma questões e uma escala likert de quatro pontos, pois é composto por vinte seis questões da escala original do MAl, sendo acrescentado um ponto na escala likert, ficando com cinco pontos (Boeckel, Wagner, Ritter, Sohne, Schein \& Grassi-Oliveira 2011).

Acresce ainda que, o IPVM é um instrumento de fácil correção, quando o resultado da aplicação apresenta pontuações elevadas, indica uma alta relação de vinculação e se for baixa pode ser um indício para a necessidade de uma intervenção nessa relação, para uma melhor assistência materna (Boeckel, Wagner, Ritter, Sohne, Schein \& Grassi-Oliveira 2011).

\section{Survey of Wellbeing of Young Children (SWYC):}

0 Survey of Wellbeing of Young Children (SWYC) é um instrumento de pesquisa norte-americano, feito em 2011 e validado em 2013, formulado para os pais responderem sobre 0 acompanhamento do desenvolvimento de seus filhos de 1 a 65 meses (Sheldrick, Henson, Neger, Merchant, Murphy \& Perrin, 2013). É composto por poucas perguntas e tem uma linguagem simples e fácil de responder, não precisa de Kit específico e está totalmente disponível na Internet. Foi aprovado pela MassHealth para formalização das diretrizes de triagem da iniciativa da saúde do comportamento infantil (Sheldrick, Henson, Merchant, Neger, Murphy\& Perrin, 2012; Sheldrick, Henson, Neger, Merchant, Murphy \& Perrin, 2013; Sheldrick \& Perin 2013; Smith, Sheldrick \& Perrin 2012).

0 SWYC foi traduzido para algumas línguas, como: 0 português, espanhol, birmanês e nepalês. 0 processo de tradução do SWYC para o português durou cerca de dois anos, foi feito pela equipe do Tufts Medical center (TMC), juntamente com os pesquisadores da Universidades Federais de Minas Gerais (UFMG) e de Santa Catarina (UFSC) (Sheldrick, Henson, Merchant, Neger, Murphy\& Perrin, 2012; Sheldrick, Henson, Neger, Merchant, Murphy \& Perrin 2013).

O SWYC foi normatizado e adaptado culturalmente em 2016, servindo para vigilância do desenvolvimento e do comportamento de crianças brasileiras, identificando precocemente riscos de alterações no desenvolvimento infantil e comportamental da criança e, a partir disso, propiciando intervenções necessárias para um saudável desenvolvimento infantil (Moreira 2016).

0 SWYC é formado por doze questionários específicos para determinadas idades $(2,4,6,9,12,15,18,24$, 30,36,48 e 60 meses), que abrangem três domínios do funcionamento infantil: 0 domínio de desenvolvimento global, o domínio emocional/social (comportamento) e o contexto familiar (Moreira, 2016; Sheldrick, Henson, Merchant, Neger, Murphy,\& Perrin 2012; Sheldrick, Henson, Neger, Merchant, Murphy, \& Perrin 2013; Whitesell, Sarche, \& Trucksess 2015).

0 domínio de desenvolvimento é formado por duas partes, um questionário sobre marcos do desenvolvimento motor, social, cognitivo e linguístico das crianças de 1 a 65 meses e a outra parte é comporta por um questionário de observação dos pais sobre interações sociais das crianças que possuem entre 18 e 35 meses (Moreira, 2016; Sheldrick, Henson, Merchant, Neger, Murphy,\& Perrin 2012; Sheldrick, Henson, Neger, Merchant, Murphy, \& Perrin 2013; Smith, Sheldrick \& Perrin 2012)

0 questionário de marcos do desenvolvimento motor, social, cognitivo e linguístico foi criado por uma equipe de onze especialistas (psicólogos, pediatras e terapeutas ocupacionais) e oito pais de crianças pequenas (de 1 a 65 meses). É formado por cinquenta e quatro questões curtas e fáceis de responder, sendo dez itens para cada idade. A soma dos escores dos 10 itens resultou no escore manual geral, que é calculado a partir dos valores dos itens (a, b1 e b2) do modelo TRI, tendo a idade correspondente a 15\% de atraso no domínio de desenvolvimento, sendo que para a utilização no Brasil, o ponto de corte de cada faixa etária foi arredondado para o próximo valor inteiro, que detecta riscos para alteração do desenvolvimento neuropsicomotor (Moreira, 2016; Sheldrick \& Perrin 2013). 


\section{INSTRUMENTOS QUE AVALIAM O VÍNCULO MATERNO E OS RISCOS DE ALTERAÇÃO NO DESENVOLVIMENTO}

INFANTIL

Com relação ao questionário de observação dos pais sobre interações sociais (POSI) de crianças de 18 e 35 meses, foi criado por um grupo multi-institucional de clínicos e psicólogos, especialistas em autismo, com a finalidade de detecção de riscos para o espectro do autismo (TEA). Foi comparado com o instrumento Modified Checklist for Autism in Toddlers (M-CHAT) para avaliar a confiabilidade e a validade, demonstrando consistência interna adequada e sensibilidade e especificidade similares ao M-CHAT (Moreira, 2016; Smith, Sheldrick \& Perrin 2012).

0 POSI é feito por sete perguntas, tendo cinco opções de respostas para cada pergunta, que tem pontuação de zero ou um, sendo as duas primeiras opções de resposta com pontuação zero e as tres restantes com pontuação um. Tem como ponto de corte, ter maior ou igual a três pontos para detecção de risco para o transtorno do espectro do autismo. É um questionario curto, rápido e de facil aplicação, leva em media 5 minutos para responder e também se encontra disponivel no site: www.theSWYC.org. (Moreira, 2016; Smith, Sheldrick \& Perrin 2012).

0 domínio sócio-emocional/comportamental também é avaliado através de um questionário, curto e fácil de ler. Foi revisado por 11 especialistas em desenvolvimento infantil e 8 pais de crianças pequenas. Foi feito para ser respondido pelos pais de acordo com a idade do seu filho, podendo ser: A Lista de Verificação de Sintomas Pediátricos do Bebê (BSPC) e o outro é a Lista de Verificação de Sintomas Pediátricos Pré-Escolares (PPSC) (Moreira 2016; Sheldrick, Henson, Merchant, Neger, Murphy \& Perrin 2012; Sheldrick, Henson, Neger, Merchant, Murphy \& Perrin 2013).

A Lista de Verificação de Sintomas Pediátricos do Bebê (BSPC), é um instrumento de triagem sócio-emocional de crianças de 1 a 18 meses, foi comparado ao Ages and Stages Questionnaires (ASQ), ao Formulário de Índice de Estresse Parental - Curto (PSI / SF) e ao Questionário de Saúde do Paciente com 2 itens (PHQ-2) (Moreira 2016; Sheldrick, Henson, Neger, Merchant, Murphy \& Perrin 2013).

A Lista de Verificação de Sintomas Pediátricos do Bebê (BSPC) é voltada para identificar riscos de distúrbios sócio-emocionais/comportamental em crianças pequenas. É um questionário breve, composto por quatorze perguntas, com pontuação de 0 a 2 pontos e avalia três fatores: irritabilidade, inflexibilidade e dificuldade com mudanças na rotina. Tem como ponto de corte brasileiro, pontuaçao igual ou acima de quatro pontos em qualquer um dos fatores citados acima, detectando de riscos de alteraçao no desenvolvimento sócioemocional/comportamental (Moreira 2016; Sheldrick, Henson, Neger, Merchant, Murphy \& Perrin 2013).

Já a Lista de Verificação de Sintomas Pediátricos Pré-Escolares (PPSC), é um instrumento de triagem sócioemocional/comportamental de crianças de 18 a 65 meses. Tem uma linguagem acessivel aos pais, é facil de pontuar e breve. Durante sua formulação foi comparado ao Child Behavior Checklist 1,5-5 anos (CBCL), Avaliação Social e Emocional Infantil (ITSEA) e Questionário Ages \& Stages: Social / Emocional (ASQ: SE) (Moreira 2016; Sheldrick, Henson, Merchant, Neger, Murphy.\& Perrin 2012).

0 PPSC é composto por 18 itens, tem pontuação entre 0 e 2 pontos, avalia fatores relacionados a internalização, externalização, sintomas de atenção, e desafios para parentagem. 0 ponto de corte do PPSC original, seria a soma dos fatores igual ou maior que 9 pontos, mas para ser utilizado no Brasil, ele foi adaptado culturamente para a soma dos fatores igual ou maior que 16 pontos, detectando riscos de alteração do desenvolvimento sócio-emocional/comportamental das crianças (Moreira 2016; Sheldrick, Henson, Merchant, Neger, Murphy.\& Perrin 2012).

0 instrumento utilizado para avaliar o contexto familiar foi criado para verificar fatores de riscos psicossociais, como abuso de álcool e drogas, depressão, conflitos parentais e insegurança alimentar que afetam o bemestar dos pais e das crianças de 1 a 65 meses. As perguntas foram retiradas dos instrumentos: Patient Health Questionnaire-2, Woman Abuse Screening Tool, Two-item Conjoint Screener. É um questionário de fácil aplicação, composto por nove itens e tem uma pontuação entre zero e três pontos. Se as respostas das quatro primeiras perguntas forem positivas, indica riscos para uso de substâncias ilícitas, se a quinta questão for positiva, indica risco para segurança alimentar, já se as questões seis e sete apresentarem soma maior ou igual a três, haverá risco de depressão materna e, para finalizar, se for positiva uma das duas questões oito e nove ou ambas, indica 
risco para violência doméstica (Moreira 2016; Sheldrick, Henson, Merchant, Neger, Murphy.\& Perrin 2012; Sheldrick, Henson, Neger, Merchant, Murphy \& Perrin 2013).

Assim, como forma de complementar esses estudos, surgiu a necessidade de aprofundar o conhecimento de dois instrumentos: Inventário de Percepção de Vinculação Materna (IPVM) e o Survey of Wellbeing of Young Children (SWYC), que são utilizados para avaliar o vínculo das mães com as crianças como também o desenvolvimento das crianças de 0 a 3 anos. Esta pesquisa será de grande relevância para a comunidade científica, assim como para a política de saúde pública, pois tem como pretensão de contribuir na prevenção e estudo de fatores que favoreçam o desenvolvimento saudável de crianças de zero a três anos.

Em consonância com o que foi dito acima, esta pesquisa tem como objetivo avaliar a consistência bibliográfica de dois instrumentos, o Inventário de Percepção de Vinculação Materna (IPVM) e o Survey of Wellbeing of Young Children (SWYC), apontando informações sobre confiabilidade e validade das suas medidas.

\section{METODOLOGIA}

Este é um estudo de revisão integrativa. Foi realizado no período de fevereiro a dezembro do ano de 2017, nas bases dos periódicos CAPES, na Biblioteca Virtual em Saúde (LILACS, MEDLINE e INDEX. PSICOLOGIA), no Pubmed e Scielo.

A revisão foi feita a partir da seguinte pergunta norteadora: Os instrumentos, Inventário de Percepção da Vincular Materna (IPVM) e Survey of Wellbeing of Young (SWYC) são confiáveis e precisos?

A pesquisa foi realizada, sem restringir o idioma, com o filtro de 10 anos e abrangendo artigos com 0 texto completo. Na pesquisa do IPVM, foi utilizado o descritor Attachment e também foi acrescido o termo "Maternal Attachment Inventory". Com relação à pesquisa referente ao SWYC, foram usados os descritores Behavioral, screening e também foi acrescido o termo SWYC. Considerando que, os instrumentos originais são internacionais e com a escassez de publicações no Brasil, a pesquisa deteve-se a utilizar os descritores em Inglês. Como critério de inclusão, foram escolhidos os artigos que se retratavam ao MAI, IPVM e SWYC e que responderam à pergunta norteadora da pesquisa. Como critério de exclusão foram retirados, reportagens, artigos repetidos e que não se referiam aos instrumentos. Todos os artigos selecionados foram lidos integralmente.

\section{RESULTADOS}

No estudo, foram encontrados o total de 68 estudos com o termo Attachemnt AND "Maternal Attachament Inventory". Durante a busca, foi feita a leitura do resumo de cada artigo, permanecendo 16 estudos que foram lidos integralmente. A pesquisa iniciou-se pela Biblioteca Virtual em Saúde (LILACS, MEDLINE e IBECS), nela foram encontrados 13 artigos, que a partir dos critérios de exclusão, serviram 12 estudos, em seguida foram localizados 10 estudos na base de dados Pubmed, todos repetidos, na Scielo foram encontrados 2 estudos, também repetidos e nos periódicos CAPES foram encontrados 43 estudos, mas somente 16 condiziam com os critérios da pesquisa, sendo 12 repetidos e 4 artigos novos que foram lidos integralmente.

Na busca referente ao SWYC, a pesquisa foi feita com o descritores Behavioral AND Screening AND SWYC. Foram encontrados 31 artigos no total, retirados os repetidos e que não se encaixavam nos critérios de inclusão ficaram 11 artigos. Também foi encontrada uma tese da Moreira (2016), totalizando 12 estudos lidos integralmente. A busca iniciou-se pela Biblioteca Virtual em Saúde (MEDLINE), nela foram encontrados 3 artigos e todos condiziam com os critérios da pesquisa, já na base de dados do Pubmed foram encontrados 4 artigos, sendo 3 repetidos e 1 para ser lido integralmente, na Scielo nenhum artigo foi encontrado e por fim foi realizada a pesquisa na base de periódicos CAPES, que foi dividida em duas etapas: A primeira foi usado os termos, Behavioral AND SWYC, foram achados 10 estudos, sendo 4 repetidos, 1 que não condizia com a pesquisa e 5 que foram lidos integralmente. A segunda parte da pesquisa foi feita com os termos, Sreenning AND SWYC, foram localizados 14 estudos, sendo 9 repetidos, 2 que não serviam e restaram 3 para serem lidos.

0 fluxograma abaixo exemplifica de forma simples a seleção dos estudos (Figura I). 
Figura I: Fluxograma de seleção e inclusão dos estudos para a revisão.

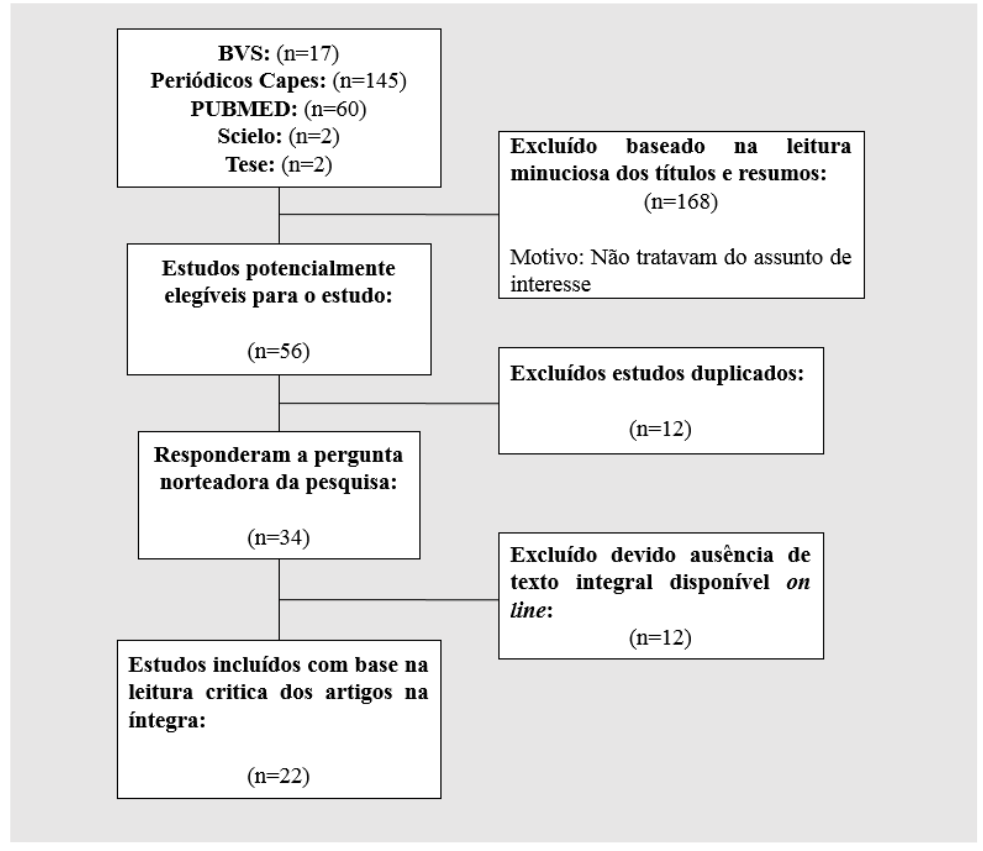

\section{DISCUSSÃO}

Pesquisas referente ao Maternal Attachment Inventory (MAI), revelaram que para o uso desse instrumento é necessário a mãe ser alfabetizada, pois se trata de um instrumento de autorrelato, referente aos sentimentos e comportamentos das mães com os seus bebês (Çinar \& Öztürk, 2013; Reay, Matthey, Sellwood, \& Scott 2011).

A grande parte dos estudos referentes ao MAl afirmaram que 0 instrumento possui uma alta fidedignidade, uma boa consistência interna e confiabilidade, servindo para avaliar o vínculo da mãe com o filho (Çinar \& Öztürk ,2013; Chen, Sung, Chen, Chang \& Lee 2013; Gharaibeh \& Hamlan 2011; lida, Horiuchi \& Porter 2012; Perrelli, Zambaldi, Cantilino \& Sougey 2014; Porter, Porter, McCoy, Bango-Sanchez, Kissel, Williams, \& Nunnewar 2015;. Sen\& Kavlak, 2012; Suetsugu, Honjo, Ikeda\& Kamibeppu, 2015; Zhang, Su, Yao, Wang, Dang, Ding, Zhu, Shao, \& Li, 2017).

Em contrapartida, Shin e Kim (2007), realizaram um estudos sobre a avaliação psicométrica do MAI coreano, perceberam inconsistência no Maternal Attachment Inventory coreano, afirmaram que 0 instrumento demonstrou fraqueza na validade e confiabilidade, apesar da maioria dos itens serem internamente consistentes. Os itens que se referem a compreensão das mães de identificar as necessidades de seus bebês, demonstraram fragilidade, pois está mais associado ao alcance de um conhecimento, sendo-os mais cognitivos do que componentes afetivos, também ressaltaram a necessidade de mais estudos para testar as propriedades psicométricas.

Por conseguinte, Boeckel et al. (2011), realizou a tradução e adaptação cultural do MAl para o IPVM, assegurou que o IPVM foi consistente para avaliar a vinculação das mães de crianças de seis anos a treze anos de idade, apresentando uma alta fidedignidade e também ressaltou a necessidade de mais pesquisas com este instrumento no Brasil que possibilitem sua validação. 
Com relação ao SWYC, é tido como um instrumento robusto, de triagem para detecção de alteração no desenvolvimento, comportamento e contexto familiar de crianças de 1 a 65 meses É extremamente útil na saúde primária e bastante necessário nas pesquisas que envolvem a saúde pública por serem úteis para identificação precoce de riscos na saúde da criança e da família (Chung et al.2016; Iyer, Dawson, Sawyer, Abdullah, Saju \& Needlman, 2017; Fierman et al. 2016; Kurtz, Levine \& Safyer 2017; Moreira 2016; Serres \& Chen 2017; Whitesell, Sarche, \& Trucksess, 2015).

Todos os questionários do SWYC, evidenciaram parâmetros adequados para uso na população brasileira, além disso, ofereceram índices aceitáveis de validade convergente e confiabilidade, fornecendo muitas vantagens em um instrumento de triagem do desenvolvimento, sendo considerado de primeiro nível, de acordo com Moreira (2016), que fez o estudo estudo normativo do "Survey of Wellbeing of Young Children (SWYC)" para crianças brasileiras.

Por fim, estudos recomendam a necessidade de mais pesquisas para comprovação da validade do SWYC, como para disseminação do uso do instrumento para além da pediatria de atenção primária (Serres \& Chen 2017; Sheldrick \& Perrin, 2013; Whitesell, Sarche, \& Trucksess 2015).

\section{CONCLUSÃO}

Diante da reduzida quantidade de estudos sobre o Maternal Attachment Inventory (MAI), como também do Inventário de Percepção de Vinculação Materna (IPVM), observou-se que são necessárias novas investigações tanto para avaliar o vínculo em todos os estágios do desenvolvimento, como também para o processo de validação do instrumento em diversas línguas, inclusive a brasileira.

Portanto, o Inventário de Percepção Vincular Materna (IPVM), é um instrumento eficaz para identificar áreas de melhoria na relação mãe-filho de crianças de seis a treze anos, podendo ser útil na triagem, atuando de forma preventiva nessa relação, como também para implementação de estratégias interventivas na saúde pública, evitando danos na relação mãe-filho e trazendo uma maior conscientização e compreensão para as mulheres sobre suas relações com os filhos.

Já o SWYC é tido como um instrumento bastante útil para vigilância do desenvolvimento neuropsicomotor, sócio emocional e de comportamento, sendo provavelmente o instrumento mais relevante para triagem comportamental de crianças pequenas e um importante sinalizador do contexto de vida das crianças.

Diante disso, concluiu-se que tanto o SWYC como o IPVM são instrumentos que podem ser utilizados em larga escalas, são úteis na atenção primária por serem de fácil acesso, viáveis, de baixo custo e de fácil aplicação. O SWYC possui eficácia técnica e precisão diagnostica, já o IPVM possui eficácia técnica, mas é necessárias mais investigações que comprovem sua precisão diagnostica.

\section{CONFLITO DE INTERESSE}

Não há conflito de interesse nesse estudo.

\section{REFERÊNCIAS BIBLIOGRÁFICAS}

Allende, L.A, Fuente, L.J.G., Rivera, F.J.P.\& García, D.F. (2016) Apego en el postparto precoz: comparación entre madres de neonatos ingresados en el Servicio de Obstetricia y en la Unidad de Cuidados Intensivos Neonatales. Enfer Glob.16(1), 295-308.

Boeckel, M.G., Wagner A., Ritter F., Sohne L., Schein S., \& Grassi-Oliveira R. (2011). Análise fatorial do inventário percepção de vinculação materna. $R$ Interam Psicol. 45(3), 439-448.

Boztepe, H., Ay A., Kerimo lu, Y. G. \& Çınar, S. (2016). Does the visibility of a congenital anomaly affect maternal-infant attachment levels? J Spec Pediatr Nurs. 21(4), 200-211.

Chen, C.J., Sung, H.C., Chen, Y.C., Chang, C.Y. \& Lee, M.S. (2013). The development and psychometric evaluation of the Chinese version of the maternal attachment inventory. J Clin Nurs.,22, 19-20: 2687-2695. 
Chung, E.K., Siegel, B.S· , Garg, A., Conroy, K·, Gross, R.S., Long, D.A., Lewis, G., Osman C.J., Jo Messito, M., Wade, R. Jr., Shonna, Y. H·, Cox, J., \& Fierman, A.H· (2016). Screening for Social Determinants of Health Among Children and Families Living in Poverty: A Guide for Clinicians. Current Problems In Pediatric And Adolescent Health Care. Elsevier BV. 46(5),135-153.

Çinar, .0̈. \& Öztürk, A.(2013). The Effect of Planned Baby Care Education Given to Primiparous Mothers on Maternal Attachment and Self-Confidence Levels. Health Care For Women International, 35(3), 320-333.

Eickmann, S.H., Emond, A.M. \& Lima, M. (2016). Evaluation of child development: beyond the neuromotor aspect. J de Ped., 92(3), 71-83.

Fierman ,A.H., Beck, A.F., Chung, E.K., Tschudy, M.M., Coker, T.R., Mistry, K.B., Siegel, B., Chamberlain, L. J., Conroy, K., Federico, S.G., Flanagan, P.J., Garg, A , Gitterman, B.A., Grace, A.M., Gross, R.S., Hole, M.K., Klass ,P. Kraft, C., Kuo, A., Lewis, G., Lobach, K.S., Long, D., Ma, C.T., Messito, M., Navsaria, D., Northrip, K.R., Osman, C., Sadof, M.D., Schickedanz, A.B., \& Cox, J. (2016). .Redesigning Health Care Practices to Address Childhood Poverty. Academic Pediatrics. Elsevier BV. 16 (3),136-146

Gharaibeh, M.K., \& Hamlan, A.M. (2011). Factors influencing maternal attachment of first-time Jordanian mothers. J Res Nurs., 17(3), 289-303.

Gürol, A., \& Polat, S. (2012). The Effects of Baby Massage on Attachment between Mother and their Infants. Asian Nurs Res. 6(1), 35-41.

lida, M., Horiuchi, S., \& Porter, S. E. (2012). The relationship between women-centred care and women's birth experiences: A comparison between birth centres, clinics, and hospitals in Japan. Elsevier BV, 28(4), 458465.

Iyer, S.N., Dawson, M.Z., Sawyer, M.I., Abdullah, N., Saju L., \& Needlman R.D.(2017). Added Value of Early Literacy Screening in Preschool Children. Clin Pediatr., 56(10), 959-963.

Kurtz, S., Levine, J., \& Safyer, M. (2017). Ask the Question: Screening for Postpartum Mood and Anxiety Disorders in Pediatric Primary Care. Elsevier BV, 47(10), 241-253.

Moreira, R.S. (2016). Triagem de atraso de desenvolvimento e de alterações de comportamento: estudo normativo do Survey of Wellbeing of Young Children (SWYC) no contexto brasileiro (Tese). Belo Horizonte: Universidade Federal de Minas Gerais - UFMG.

Perrelli, J.G.A., Zambaldi, C.F., Cantilino A., \& Sougey, E.B.(2014). Instrumentos de avaliação do vínculo entre mãe e bebê. Rev Paul Pediatr., 32(3), 257-265.

Porter, L. S., Porter, B.O., McCoy, V., Bango-Sanchez, V., Kissel, B., Williams, M., \& Nunnewar, S.(2015). Blended Infant Massage-Parenting Enhancement Program on Recovering Substance-Abusing Mothers Parenting Stress, Self-Esteem, Depression, Maternal Attachment, and Mother-Infant Interaction. Asian Nursing Research. Elsevier BV, 9(4), 318-327.

Reay, R., Matthey, S., Ellwood, D., \& Scott M.(2011). Long-term outcomes of participants in a perinatal depression early detection program. Journal Of Affective Disorders. Elsevier BV. 129(1-3), 94-103 .

Sen, S., \& Kavlak, 0. (2012) Transgenerational attachment in Manisa, Contemp Nurse. 41(1), 126-132.

Serçeku, P., \& Ba kale, H. (2016) Effects of antenatal education on fear of childbirth, maternal self-efficacy and parental attachment. Midwifery. 34, 166-172.

Serres, S.K. \& Chen, C. (2017). Utilizing trauma admissions as an opportunity to identify developmental and behavioral concerns. The American Journal Of Surgery, Elsevier BV. 214(4), 661-665.

Sheldrick, R.C., Henson, B.S., Merchant, S., Neger, E.N., Murphy, J.M.\& Perrin, E.C. (2012).The Preschool Pediatric Symptom Checklist (PPSC): Development and Initial Validation of a New Social/Emotional Screening Instrument. Acad Pediatr., 12(5), 456-467.

Sheldrick, R.C., Henson, B.S.; Neger, E.N.; Merchant S., Murphy, J.M. \& Perrin E.C. (2013). The Baby Pediatric Symptom Checklist: Development and Initial Validation of a New Social/Emotional Screening Instrument for Very Young Children. Acad Pediatr.13(1), 72-80. 


\section{PSICOLOGÍA, INFANCIA Y EDUCACIÓN}

Sheldrick, RC., \& Perrin, E.C. (2013).Evidence-Based Milestones for Surveillance of Cognitive, Language, and Motor Development. Acad Pediatr. 13(6), 577-586.

Shin, H. \& Kim, Y.H. (2007). Maternal Attachment Inventory: psychometric evaluation of the Korean version. $J$ Adv Nurs., 59(3), 299-307.

Smith, N.J., Sheldrick, R.C. \& Perrin, E.C. (2012) An Abbreviated Screening Instrument for Autism Spectrum Disorders. Infant Ment Health J. 34(2), 149-155.

Smith, T.R.(2016). Developmental Surveillance and Screening in the Electronic Health Record. Pediatric Clinics Of North America. Elsevier BV. 63(5), 933-943.

Suetsugu, Y., Honjo, S., Ikeda M. \& Kamibeppu, K. (2015). The Japanese version of the Postpartum Bonding Questionnaire: Examination of the reliability, validity, and scale structure. J Psychosom Res. 79(1), 55-61.

Weitzman, C.\& Wegner, L. (2015). Promoting Optimal Development: Screening for Behavioral and Emotional Problems. Pediatrics, 135(2), 384-95.

Whitesell, N.R., Sarche, M., \& Trucksess, C. (2015). The survey of well-being of young children: results of a feasibility study with american indian and alaska native communities. Infant Ment Health J., 36(5), 483-505.

Zhang, H., Su Q., Yao D., Wang, S., Dang, S., Ding, D., Zhu, Z·, Shao, S., \& Li H. (2017). Prolactin, a potential mediator of reduced social interactive behavior in newborn infants following maternal perinatal depressive symptoms. J Affect Disord., 215, 274- 280. 
